\title{
PSYCHOPHYSICAL EVIDENCE FOR TWO ROUTES TO SUPPRESSION BEFORE BINOCULAR SUMMATION OF SIGNALS IN HUMAN VISION
}

\author{
D. H. BAKER, T. S. MEESE* AND R. J. SUMMERS \\ Life and Health Sciences, Aston University, Aston Street, Birmingham \\ B47ET, UK
}

\begin{abstract}
Visual mechanisms in primary visual cortex are suppressed by the superposition of gratings perpendicular to their preferred orientations. A clear picture of this process is needed to (i) inform functional architecture of image-processing models, (ii) identify the pathways available to support binocular rivalry, and (iii) generally advance our understanding of early vision. Here we use monoptic sine-wave gratings and cross-orientation masking (XOM) to reveal two crossoriented suppressive pathways in humans, both of which occur before full binocular summation of signals. One is a within-eye (ipsiocular) pathway that is spatially broadband, immune to contrast adaptation and has a suppressive weight that tends to decrease with stimulus duration. The other pathway operates between the eyes (interocular), is spatially tuned, desensitizes with contrast adaptation and has a suppressive weight that increases with stimulus duration. When cross-oriented masks are presented to both eyes, masking is enhanced or diminished for conditions in which either ipsiocular or interocular pathways dominate masking, respectively. We propose that ipsiocular suppression precedes the influence of interocular suppression and tentatively associate the two effects with the lateral geniculate nucleus (or retina) and the visual cortex respectively. The interocular route is a good candidate for the initial pathway involved in binocular rivalry and predicts that interocular cross-orientation suppression should be found in cortical cells with predominantly ipsiocular drive. (c) 2007 IBRO. Published by Elsevier Ltd. All rights reserved.
\end{abstract}

Key words: human vision, psychophysics, cross-orientation inhibition, contrast gain control, masking, binocular rivalry.

Masking is the psychophysical phenomenon whereby the addition of a 'mask' stimulus to a target image causes information in the target to be lost to the observer. There are probably several different processes involved in the various masking phenomena in the literature (Harmon and Julesz, 1973; Legge and Foley, 1980; Morrone et al., 1983; Born and Tootell, 1991; Foley, 1994; Olzak and Thomas, 1999; Macknik and Martinez-Conde, 2004; Meese and Holmes, 2007), but one which is thought to underlie several of them is suppression. Of particular interest here is

*Corresponding author. Tel: +44-0-121-204-4130; fax: +44-0-121-2044048.

E-mail addresses: t.s.meese@aston.ac.uk (T. S. Meese); bakerdh@ aston.ac.uk (D. H. Baker); r.j.summers@aston.ac.uk (R. J. Summers).

Abbreviations: LGN, lateral geniculate nucleus; RMS, root mean square; S.E., standard error; V1, visual area 1 ; XOM, cross-orientation masking; XOS, cross-orientation suppression. the variant known as cross-orientation suppression (XOS): a cell's response to a stimulus at its preferred orientation is reduced by the superposition of a mask stimulus at another orientation (Morrone et al., 1982; Bonds, 1989). Early work supposed that XOS is caused by intra-cortical inhibition (Morrone et al., 1987; Heeger, 1992), but recent studies of cat physiology have challenged this view. For example, mask stimuli that drift or flicker too quickly to excite most cortical cells will nevertheless produce XOS, implying precortical involvement (Freeman et al., 2002; Sengpiel and Vorobyov, 2005). Possible origins include suppressive interactions in the lateral geniculate nucleus (LGN) (Levick et al., 1972; Bonin et al., 2005), saturation in the retina or LGN (Li et al., 2006; Priebe and Ferster, 2006; Smith et al., 2006) and depression at the thalamo-cortical synapse (Freeman et al., 2002). The last two accounts are applicable only when the mask and test are presented to the same eye (monoptic presentation) and overlap in space and time. However, in cat at least, XOS is not a purely ipsiocular process because when an oriented grating and crossoriented mask are presented to different eyes (dichoptic presentation), suppression is evident in striate cells (Sengpiel et al., 1995; Walker et al., 1998; Li et al., 2005; Sengpiel and Vorobyov, 2005). Although interocular suppression has been found in the LGN (e.g. Sanderson et al., 1969; Murphy and Sillito, 1989; Felisberti and Derrington, 1999) a cortical origin for dichoptic XOS seems likely since it is diminished when the GABA antagonist bicuculline is used to block intracortical inhibition (Sengpiel and Vorobyov, 2005). These results suggest that at least two mechanisms underlie XOS in cat: a cortical interocular mechanism, and a pre-cortical ipsiocular mechanism.

Cross-orientation masking (XOM) is a phenomenon observed at the behavioral level and is thought to be a consequence of the neural process of XOS (Foley, 1994; Meese and Hess, 2004). With appropriate stimulation, these psychophysical effects can be substantial, raising contrast detection threshold by more than a factor of 4 (Meese and Holmes, 2007). If the dual pathways for XOS in cat are also to be found in primate, then we might expect eye of origin of mask and test gratings to influence human performance. However, most previous studies of XOM have used binocular presentation (e.g. Foley, 1994; Meese and Holmes, 2007), which one might suppose combines masking influences from both within and between the eyes (though see Discussion). Few studies have attempted to separate the two, and those that have (e.g. Legge, 1979; Levi et al., 1979; Meese and Hess, 2004) did not provide a detailed comparison of monoptic and dichoptic XOM func- 
tions. We address this here with the specific aim of investigating the number of pathways involved in human XOM.

\section{EXPERIMENTAL PROCEDURES}

\section{Equipment and observers}

Stimuli were displayed on a Clinton Monoray monitor with a frame rate of $120 \mathrm{~Hz}$ using a Cambridge Research Systems ViSaGe stimulus generator controlled by a desktop computer and were viewed through a mirror stereoscope (Cambridge Research Systems, Rochester, Kent, UK). A central fixation point was present throughout, and stimuli were displayed in the center of a circular aperture with a diameter of $9^{\circ}$ and a dark surround, at an optical viewing distance of $57 \mathrm{~cm}$. Mean luminance of the central display region was $30 \mathrm{~cd} / \mathrm{m}^{2}$ after attenuation by a neutral density filter. The display was gamma corrected to ensure linearity over the full contrast range, and a frame interleaving technique $(60 \mathrm{~Hz}$ per image) was used for all conditions, allowing the contrasts of the mask and test stimuli to be varied independently using lookup tables.

The three observers were all psychophysically experienced and wore their normal optical correction.

\section{Stimuli}

Mask and test stimuli were circular patches of sinusoidal grating, spatially modulated by a raised cosine window (see inset to Fig. 2 ), with a central plateau $3^{\circ}$ in diameter. Test gratings were always horizontal and had a spatial frequency of $1 \mathrm{c} /{ }^{\circ}$. Two different cross-oriented mask gratings were used. One was a vertical grating (oriented perpendicular to the test) with a spatial frequency of $1 \mathrm{c} /{ }^{\circ}$. The other was an oblique grating $\left(-45^{\circ}\right)$ with a spatial frequency of $3 \mathrm{c} /{ }^{\circ}$. The first arrangement is a canonical stimulus for XOM and uses mask and test spatial frequencies that are known to produce large effects (Meese and Holmes, 2007). The second extends the stimulus space in spatial frequency and orientation and helps place the present study within a larger body of ongoing psychophysical work (Meese and Holmes, 2002; Meese, 2004; Meese and Hess, 2004; Meese et al., in press).

There were two ocular conditions for each type of mask. In the monoptic condition, the mask and test were presented to the same eye and the other eye was presented with mean luminance. In the dichoptic condition the mask and test were presented to different eyes. (A third, 'half-binocular,' condition is described in the Discussion.) On each trial, the spatial phase of the mask and the test was randomly selected from one of four values $\left(0,90,180\right.$ or $\left.270^{\circ}\right)$ to homogenize local luminance adaptation. Stimulus contrast is expressed as Michelson contrast in percent, given by $c=100\left(\mathrm{~L}_{\max }-\mathrm{L}_{\min }\right) /\left(\mathrm{L}_{\max }+\mathrm{L}_{\min }\right)$.

In experiments I and II, detection thresholds were measured for all four mask/test configurations. These experiments explored the effects of stimulus duration $(25-400 \mathrm{~ms})$ and mask contrast $(0 \%-45 \%)$, respectively. The mask and test were always superimposed in time and had the same duration. Experiment III used an adaptation paradigm, in which the mask eye was presented with an adapting pattern for $2 \mathrm{~min}$. This was a vertical $1 \mathrm{c} /{ }^{\circ}$ grating with contrast of either $0 \%$ or $50 \%$. To evenly distribute local luminance adaptation, the phase of the adaptor was shifted by $90+x^{\circ}$ every $100 \mathrm{~ms}$, where $x$ was a value randomly selected from the range $0-180^{\circ}$. Top-up adaptation periods of $6 \mathrm{~s}$ occurred before each trial and were followed by a blank period of $500 \mathrm{~ms}$ before the first test interval.

In all three experiments, monoptic detection thresholds were also gathered in the absence of a mask (a no-mask control condition). In experiment II, binocular detection thresholds were also measured for a mask contrast of $0 \%$. This was to help specify some of the model parameters as described in Appendix A.

\section{Procedure}

Subjects were seated in a dark room with their heads in a support to which the stereoscope was attached. A two-interval forcedchoice (2IFC) procedure was used to estimate detection thresholds (interstimulus interval $=500 \mathrm{~ms}$ ). One interval contained only the mask grating, and the other contained the mask plus the test grating. Subjects indicated which interval contained the test grating by pressing one of two mouse buttons and auditory feedback indicated correctness of response. Stimulus conditions were blocked by mask contrast level, duration and mask type (i.e. $1 \mathrm{c} /{ }^{\circ}$ or $3 \mathrm{c} /{ }^{\circ}$ ). Within each block, the monoptic and dichoptic conditions were randomly interleaved across trials. For each condition, a pair of three-down, one-up staircases was used (Wetherill and Levitt, 1965), where the target contrast was decreased after three consecutive correct responses and increased after a single incorrect response. For each staircase, the contrast step-size began at 12 $\mathrm{dB}$ (a factor of 4 ), but decreased to $3 \mathrm{~dB}$ (a factor of $\sqrt{ } 2$ ) after the initial reversal in staircase direction. Each staircase terminated after 12 subsequent reversals in direction (typically about 47 trials), and only the data gathered during this stage were used in the analysis. In experiments I and II the two staircases in a pair tracked thresholds for complementary conditions across eye and were randomly interleaved. In experiment III, just one eye was adapted on each day of experimentation. The mask was presented to the adapted eye, and the test was presented to either the same eye (monoptic) or the other eye (dichoptic). In all experiments, results were similar for the complementary conditions and so the data for each condition of interest (no-mask, monoptic mask, dichoptic mask) were collapsed across the eye tested. Each observer repeated the experiment four times, and thresholds ( $75 \%$ correct) and standard errors (S.E.) were calculated by probit analysis after collapsing the data across replication. This produced individual threshold estimates for each observer based on about 375 trials.

The block length of experimental sessions (a single mask contrast) varied across experiments and conditions (e.g. stimulus duration), but was typically between 5 and $20 \mathrm{~min}$. Thus, we cannot rule out the possibility that there was a small buildup of adaptation to the briefly presented ( $\leq 400 \mathrm{~ms})$ masks. Our use of a blocked design means that high contrast masks are unlikely to have interfered with lower contrast masks (as they might in an intermixed design), but it is possible that the effective masking contrast might have been attenuated, similar to compressing the mask contrast axis slightly in Fig. 5. A further advantage of the blocked design over the intermixed design is that observers were able to attend to the appropriate visual cue for each condition, thus reducing potentially confounding effects of uncertainty (e.g. the visual cue for a horizontal grating target without a mask can be very different from that in the presence of a high contrast vertical mask).

\section{RESULTS}

\section{Qualitative model predictions}

Four possible arrangements for XOS are shown in Fig. 1, where the models are devised to operate around psychophysical detection threshold. We consider the situations where the test grating is presented to one eye (it does not matter which, hence icons are shown for both eyes) and the mask is also presented to only one eye which is either the same (monoptic) or different (dichoptic) from the test. In principle, masking from monoptic and dichoptic stimuli could be due to a common mechanism at a purely binocular site. This is shown in Model 1 (Fig. 1a), where XOS is placed after binocular summation. In this case, the contrast 


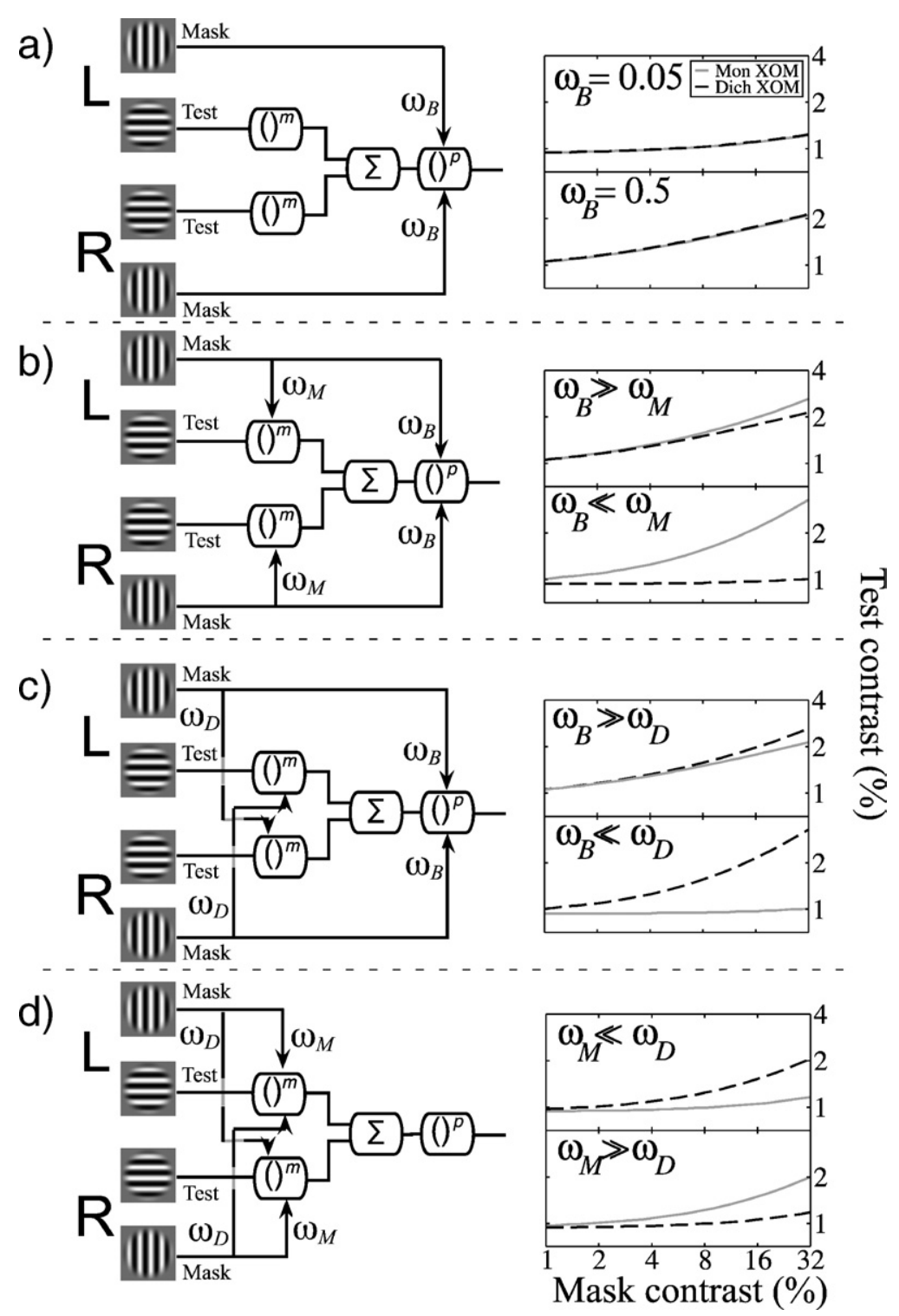

Fig. 1. Schematic diagrams (left) and ordinal predictions (right) for the four different logical arrangements of XOS. In each model, the target (test) is a patch of horizontal grating presented to either the left or right eye. The mask is a patch of vertical grating presented to either the same (monoptic) or the different (dichoptic) eye from the target. The $\Sigma$ symbol denotes linear summation of left and right eye responses to the test contrast $(L$ and R). In Model 1 (a), XOS is placed after binocular summation, in Model 4 (d) it is placed before binocular summation and in Models 2 and 3 (b and $c$ ) it is placed in both locations but in different arrangements. The parameters $\omega_{M}, \omega_{D}$ and $\omega_{B}$ are suppressive weights, for monoptic, dichoptic and binocular sites of XOS respectively. Illustrative values for these weights are shown in the panels on the right. Where $\omega$ is not referenced it was set to zero. The panels on the right show that as mask contrast increases, then more test contrast is needed to detect the target (i.e. XOM occurs). Note that the different models make different predictions about whether monoptic or dichoptic masking is the greater, but that in no case does the order of the masking functions change with mask contrast.

detection threshold of the test grating is raised in exactly the same way (curves in the panels on the right), regardless of whether the test is presented to the same or different eye from the mask. On the other hand, if XOM involves two (or more) different mechanisms, we should not expect monoptic and dichoptic masking functions to superimpose (Fig. 1b-d). For example, if the monoptic influence is placed before binocular summation, then monoptic masking is constrained to be greater than dichoptic masking (Model 2; Fig. 1b). There are corresponding constraints when the dichoptic influence is placed before binocular summation (Model 3; Fig. 1c). The most flexible arrange- ment is when both influences are placed before binocular summation, in which case either type of masking can be greater, depending on the relative weights of XOS from within $\left(\omega_{M}\right)$ and between $\left(\omega_{D}\right)$ the eyes (Model 4; Fig. 1d). Our first two experiments investigate whether human vision operates with any of the constraints produced by these configurations.

\section{Experiment I: temporal dependencies of XOM}

Fig. 1 establishes the logic of our approach, but before reporting the contrast-masking functions (experiment II) 
we first consider the effects of stimulus duration at the high contrast end of these functions (far right of the plots in Fig. 1). We do this for several reasons. First, stimulus duration is known to be important for some suppressive phenomena (e.g. see Solomon et al., 2006). Second, the temporal characteristics of the masking process will help to constrain the association between our systems analysis and the physiological underpinnings. Third, increasing the number of stimulus conditions raises the probability of being able to decide between Model 4 and the others. Fourth, the results from this experiment can be used to fix the model parameters used in experiment II.

Fig. 2 shows the effect of stimulus duration on detection thresholds for a horizontal $1 \mathrm{c} /{ }^{\circ}$ patch of grating. For all three observers (different rows), detection thresholds mea-

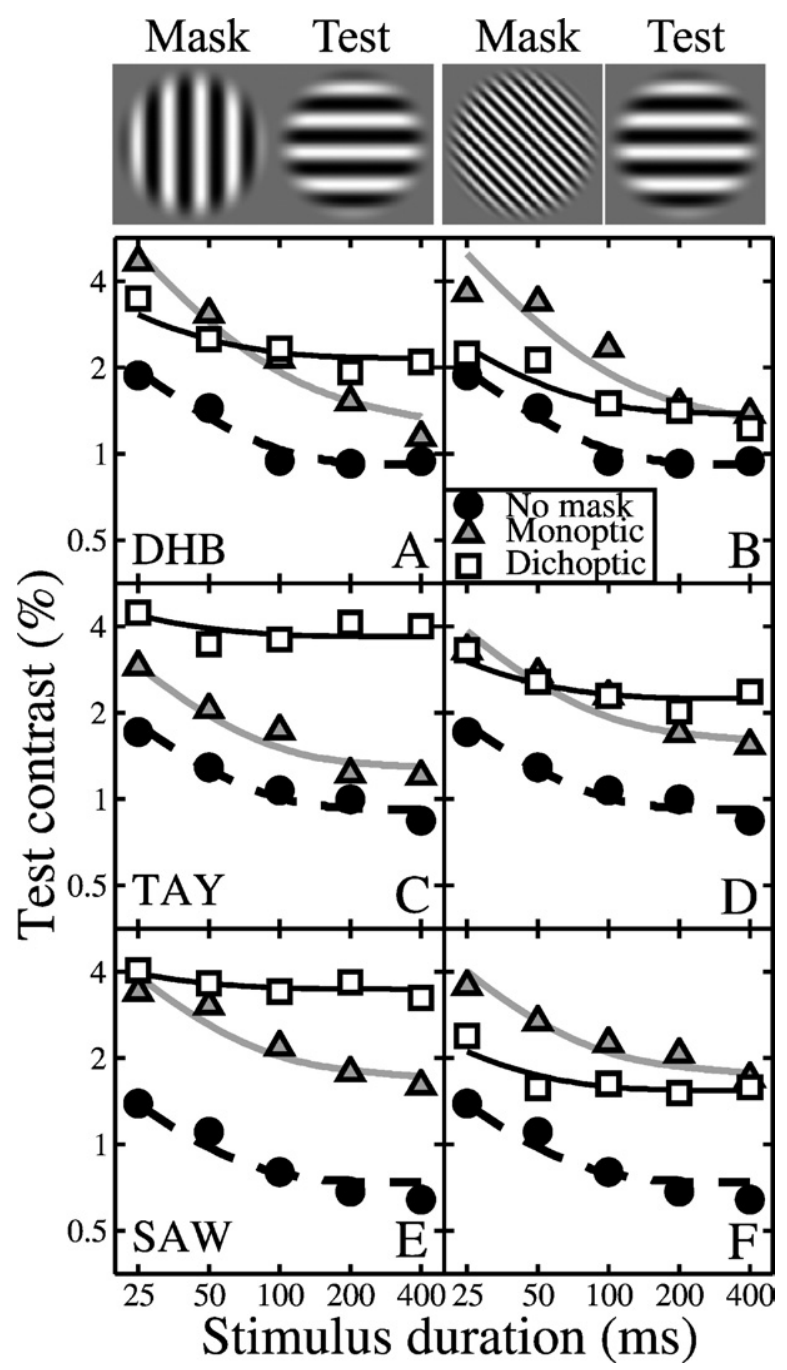

Fig. 2. Detection thresholds as functions of stimulus duration (mask contrast $=45 \%$ ) for three observers (different rows) and two mask configurations (different columns). Error bars ( $\pm 1 \mathrm{SE}$ ) were all smaller than symbol size. The no-mask thresholds (circles) are duplicated across panels for each observer. Monoptic (triangles) and dichoptic (squares) masking functions are very different, tending to converge and diverge with the no-mask thresholds respectively. The diversity of these results rejects Models 1, 2, and 3, but is well fit by Model 4 (Fig. 1d) as described in the text. sured without a mask (circles, duplicated across columns) decrease with stimulus duration, quite sharply at first, but then more gently, approaching a plateau (Legge, 1978; Georgeson, 1987; Luntinen et al., 1995). Results are also shown for a superimposed mask stimulus that was either a vertical $1 \mathrm{cl}^{\circ}$ grating (left column) or a left oblique $3 \mathrm{c} /{ }^{\circ}$ grating (right column). Like the baseline detection thresholds (circles), monoptic (triangles) and dichoptic (squares) masking functions also tend to decrease with stimulus duration, at least over the initial part of the function. Crucially, the two functions (triangles and squares) do not superimpose, providing strong evidence against the idea that XOM involves a single mechanism placed after binocular summation (Model 1).

For Models 2 and 3, the potency of one type of masking (monoptic or dichoptic) must be consistently greater than the other. This is shown in the right of Fig. 1, where the independent variable is mask contrast, though the logic is identical for monotonic masking functions of stimulus duration. In the experiment, however, there is no evidence for such an orderly arrangement. Instead, the stronger masking switches between monoptic and dichoptic stimulus conditions across duration (compare triangles and squares in Fig. 2a), mask-type (compare the triangles and squares in Fig. 2e, with those in Fig. 2f) and observer (compare the triangles and squares in Fig. 2b, with those in Fig. 2d). Put another way, neither triangles nor squares are consistently higher than the other in Fig. 2. Clearly, human vision does not operate under the constraints of Models 2 or 3 either.

The results in Fig. 2 extend previous findings of observer differences using related stimuli (Meese and Hess, 2004; Petrov and McKee, 2006), but we now see that their diversity overlies a systematic trend with stimulus duration. The no-mask functions (circles) tend to converge with the monoptic functions (triangles), but to diverge with the dichoptic functions (squares). This is seen more readily in Fig. 3, where the masking effects (threshold elevation) are shown separately for the two ocular conditions (different panels) after normalizing to unity at the shortest stimulus duration. Plotted this way, we see how masking varies with stimulus duration, where points above and below unity indicate increases and decreases respectively. In the dichoptic condition (right panel), there is a strong tendency for masking to increase with stimulus duration. This was confirmed by linear regression, which produced a positive slope for all six functions (the solid line is their average). In the monoptic condition (left panel), the results were slightly less clear. The overall trend was for masking to decrease with stimulus duration (solid line), though for one observer (SAW; see Fig. 2e and f) there was little or no effect. Nevertheless, it is clear that monoptic and dichoptic masking has different dependencies on stimulus duration, suggesting that different mechanisms are involved.

This point is emphasized further in Fig. 4, where the results for the monoptic (triangles) and dichoptic (squares) conditions have been normalized to the monoptic results across stimulus duration to simplify the comparison between the conditions (the diamonds will be described later 


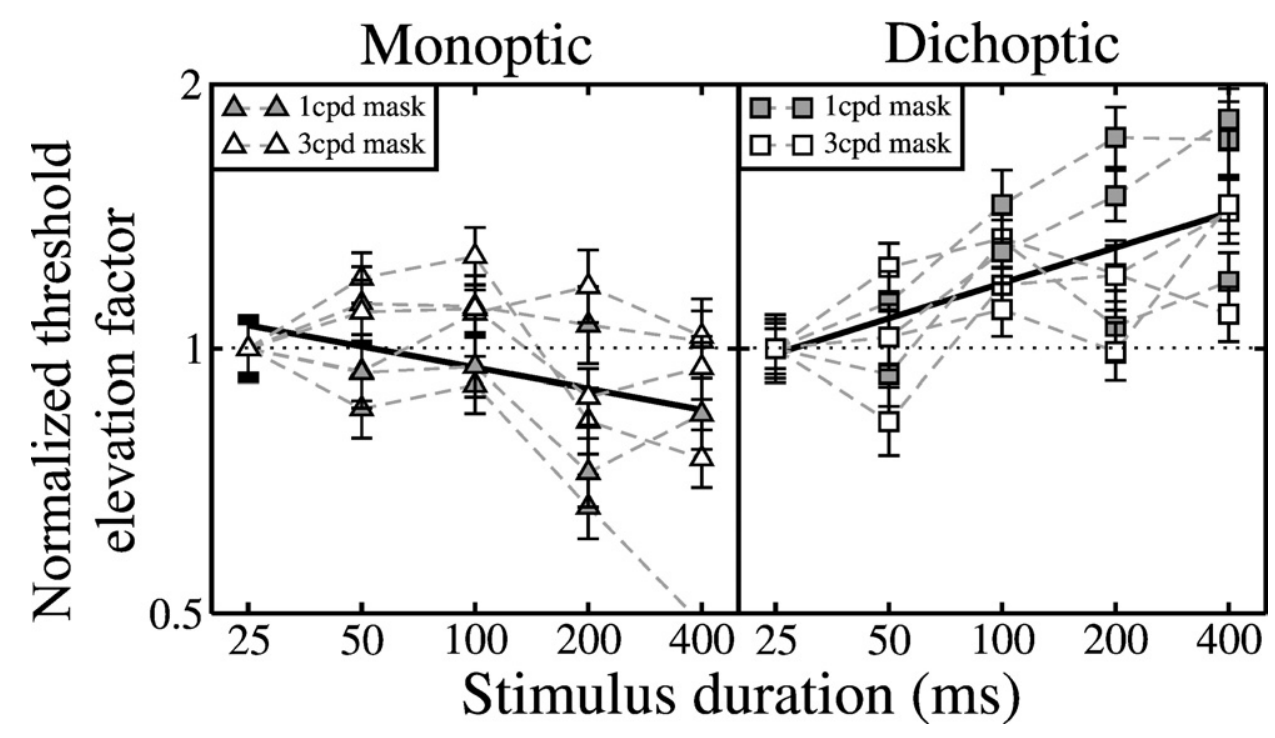

Fig. 3. Effect of stimulus duration on threshold elevation factor for monoptic (left) and dichoptic (right) XOM, derived from the results in Fig. 2. Threshold elevation was determined by calculating the ratio of contrast detection threshold with and without a mask. These functions were then normalized to unity at the shortest stimulus duration $(25 \mathrm{~ms})$. Solid and open symbols are for the $1 \mathrm{c} /{ }^{\circ}$ and $3 \mathrm{c} /{ }^{\circ}$ masks respectively (from the left and right columns in Fig. 2). The solid lines are the average linear regressions for the six functions in each panel. The horizontal dotted lines indicate the locus of null effect.

in the Discussion). Dichoptic masking increases with stimulus duration against monoptic masking (squares versus triangles) for both mask types (left and right columns) and for all three observers (different rows). But whether dichoptic masking exceeds monoptic masking (gray shading) for none, part, or all of the function depends upon both of these factors. Clearly, isolated comparisons across monoptic and dichoptic masking conditions are likely to appear inconsistent and/or depend on the details of the stimuli used (Meese and Hess, 2004; Petrov and McKee, 2006). Thus, one cannot simply characterize XOM in terms of whether the monoptic or dichoptic variety is the more potent. Much more systematic is how the levels of masking for the two ocular conditions vary against each other along the stimulus dimension of interest, be that duration (Figs. 3 and 4) or spatial frequency/orientation of the mask, as can be seen by looking ahead to Fig. 6 .

As we show in the next subsection, the one model that has not been rejected (Model 4) can accommodate all of these results. An important architectural feature of this model is that XOS from monoptic and dichoptic sources occurs before binocular summation (Fig. 1d). We describe the quantitative details below.

\section{XOM precedes binocular summation: model details}

The generic stimulus-response equation used to generate the predictions in Fig. 1 is:

$$
\begin{aligned}
& \text { resp }_{\text {test }}=\left[\frac{L^{m}}{1+\left(\omega_{M} X_{L}\right)+\left(\omega_{D} X_{R}\right)}+\frac{R^{m}}{1+\left(\omega_{M} X_{R}\right)+\left(\omega_{D} X_{L}\right)}\right]^{p} \\
& {\left[1+\left(\omega_{B} X_{L}\right)+\left(\omega_{B} X_{R}\right)\right] }
\end{aligned}
$$

where $L$ and $R$ are the test contrasts in the left and right eyes, $X_{L}$ and $X_{R}$ are the mask contrasts in the left and right eyes and $\omega_{M}, \omega_{D}$ and $\omega_{B}$ are the weights of suppression for monoptic, dichoptic and binocular masks respectively. This model equation is derived from the binocular summation model of Meese et al. (2006), extended here to accommodate the impact of XOS at various loci (see Fig. 1). However, for simplicity (to reduce the number of free parameters), the model is restricted to operate only around the detection threshold of the target and therefore describes only the accelerating part of the contrast transducer. This means it lacks the self-suppression terms on the denominator that produce the familiar sigmoidal contrast response seen at the cellular level in striate cortex (e.g. Ohzawa et al., 1985) and inferred by psychophysical pedestal masking (e.g. Legge and Foley, 1980). Further analysis (not shown) using a more elaborate version of the model that included these extra features confirmed our conclusions here.

Of the four model configurations embodied by equation 1 (Fig. 1), the only one that is viable is Model 4 (see above). For this model, the binocular response to a test grating reduces to:

$$
\operatorname{resp}_{\text {test }}=\left[\frac{L^{m}}{1+\left(\omega_{M} X_{L}\right)+\left(\omega_{D} X_{R}\right)}+\frac{R^{m}}{1+\left(\omega_{M} X_{R}\right)+\left(\omega_{D} X_{L}\right)}\right]^{p}
$$

The exponents $m$ and $p$ describe the accelerating contrast response at monoptic and binocular signal stages respectively. Their precise values are unimportant for the logic of our arguments (e.g. we found similar results with $m=p=1$ ), but we include them here for completeness. Their values were derived from the psychophysical data as described in Appendix A, giving $m=1.3$ and $p=2.8$, and are in close agreement with those estimated in earlier work at detection threshold (Georgeson and Meese, 2005). 


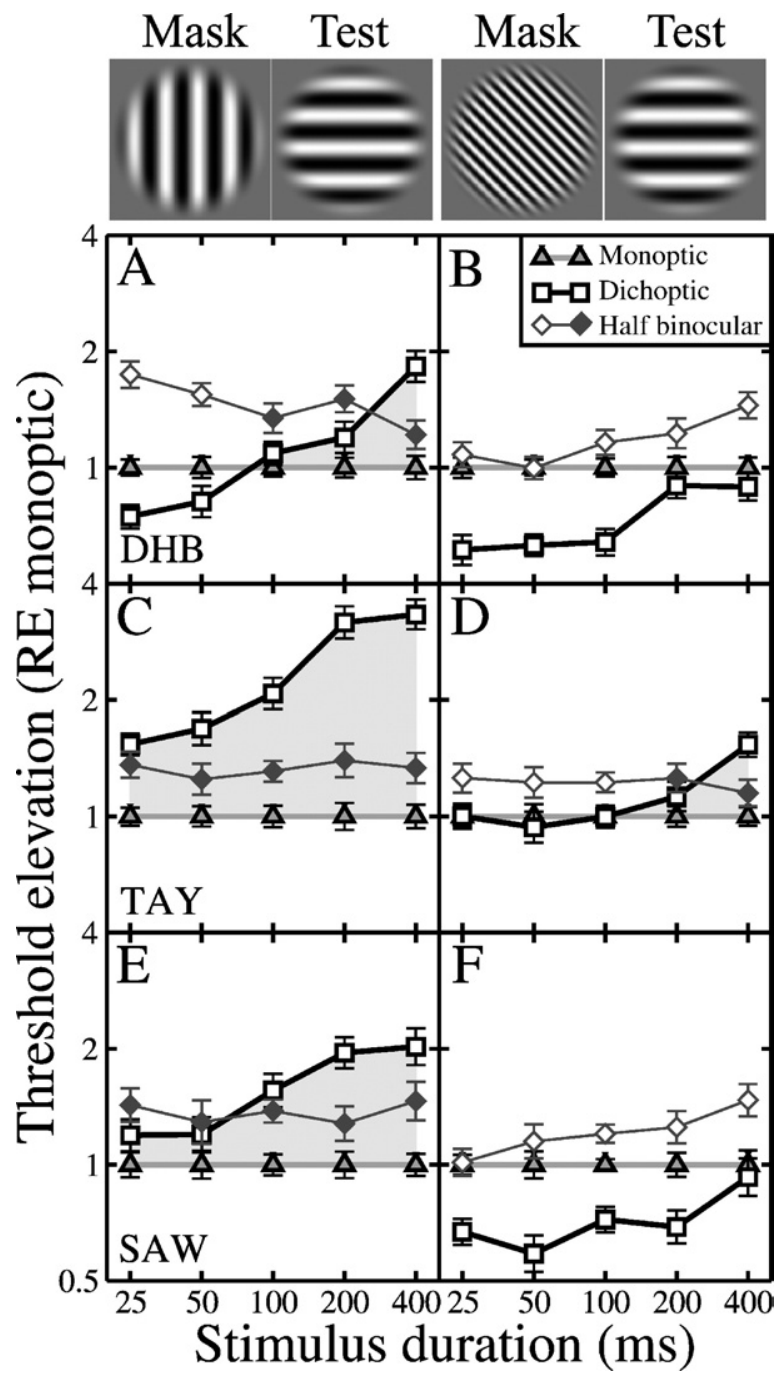

Fig. 4. Effect of stimulus duration on dichoptic and half-binocular masking relative to monoptic masking. In all cases, the severity of dichoptic masking (squares) increases against monoptic masking (triangles). The gray shading indicates the regions where dichoptic $\mathrm{XOM}>$ monoptic XOM. The diamonds are for a half-binocular condition performed at the same time (blocked) with the other conditions and are described in the Discussion. The diamonds are filled at stimulus durations where squares $>$ triangles, and are open otherwise. Note the close conjunction between the filled diamonds and the shaded regions.

Following common practice, we assume that the stimulus is detected when the difference between the model's responses to the two intervals (assumed to be zero in the null interval) is greater than or equal to some criterion level, $k$. This is a free parameter related to the standard deviation of late additive noise and sets the model observer's overall sensitivity.

Experiment I revealed three temporal dependencies: monoptic XOS, dichoptic XOS and basic detection thresholds. To model these we convert the two suppressive weights $\left(\omega_{M}\right.$ and $\left.\omega_{D}\right)$ to functions of stimulus duration, and introduce an excitatory function of stimulus duration on the numerator of the model equation. For brevity, we first rewrite equation 2 as

$$
r e s p_{\text {test }}=\left(\text { stage } 1_{\text {test,left }}+\text { stage } 1_{\text {test,right }}\right)^{p}
$$

allowing us to write

$$
\text { stage } 1_{\text {test, left }}=\frac{f_{E}(t) L^{m}}{1+\left(\omega_{M}(t) X_{L}\right)+\left(\omega_{D}(t) X_{R}\right)}
$$

for the left eye response, where $X_{L}$ and $X_{R}$ are the monoptic (left) and dichoptic (right) mask contrasts. Baseline (no-mask) detection thresholds decreased with stimulus duration (circles in Fig. 2), consistent with temporal integration. Here we find that this is well described by an inverted exponential function of the form: $f_{E}(t)=1-e^{-t / \alpha}$, where $t$ is stimulus duration in milliseconds, and $\alpha$ is a free parameter. No doubt, the underlying process is more complicated than this, possibly involving probability summation over time (Tolhurst, 1975; Watson, 1979) and multiple detectors with various impulse responses (Legge, 1978; Georgeson, 1987). Nevertheless, our descriptive approach is adequate for our present purposes and provides a good fit to the range of stimulus durations used here (e.g. dashed curves in Fig. 2).

We also achieved a very good account of dichoptic XOM using the same temporal integration function as for detection. That is, $\omega_{D}(t)=W_{D} f_{E}(t)$, where $W_{D}$ is a scaling parameter. The divergence between the baseline and dichoptic masking functions (dashed and solid dark curves in Fig. 2) occurs in the model because the masking term dominates the denominator only at longer stimulus durations (i.e. only when it is much greater than the saturation constant of unity in equation 3 ). Note that because the temporal dependencies on the numerator and denominator of equation 3 are identical the divergence between these curves is asymptotic.

The functional form of monoptic masking was different from dichoptic masking (Fig. 3), and is well approximated by $\omega_{M}(t)=W_{M} t^{\eta}$. The free parameter $\eta$, determines whether monoptic masking increases $(\eta>0)$ or decreases $(\eta<0)$ with stimulus duration. We achieved the best fits with $\eta<0$ for all three observers.

The basic model has five free parameters: the suppressive weights $\left(W_{M}, W_{D}\right)$, the temporal parameters $(\alpha$, $\eta$ ) and the sensitivity parameter $(k)$. However, it was fit simultaneously to results for both mask types, giving two independent values for each of $W_{M}$ and $W_{D}$. This gives seven free parameters for five masking functions for each observer. The model was fit to the data using a downhill simplex algorithm to minimize the root mean square (RMS) error in $\mathrm{dB}\left(20 \log _{10}(c)\right)$ between model and data. The fits are shown by the curves in Fig. 2 for experiment I and are very good. The parameter values and a figure of merit (RMS error) are shown in Table 1.

\section{Experiment II: cross-orientation contrast masking}

We tested the model further by exploring the effects of mask contrast at a stimulus-duration of $200 \mathrm{~ms}$ (Fig. $5 \mathrm{c}-\mathrm{h}$ ). As predicted (Fig. 1), masking increased with the contrast of all types of mask. But in no case did the functions superimpose, confirming our earlier rejection of Model 1. 
Table 1. Quality of fit (RMS error) and parameter values for the modeling in experiment I

\begin{tabular}{lllllllll}
\hline Observer & RMS error $(\mathrm{dB})$ & $k$ & $\alpha$ & $\eta$ & $W_{M}\left(1 \mathrm{c} /{ }^{\circ}\right.$ mask $)$ & $W_{M}\left(3 \mathrm{c} /{ }^{\circ}\right.$ mask $)$ & $W_{D}\left(1 \mathrm{c} /{ }^{\circ}\right.$ mask $)$ & $W_{D}\left(3 \mathrm{c} /{ }^{\circ} \mathrm{mask}\right)$ \\
\hline DHB & 0.96 & 0.75 & 51.2 & -0.47 & 0.23 & 0.22 & 0.04 & 0.02 \\
TAY & 0.72 & 0.77 & 44.9 & -0.15 & 0.03 & 0.06 & 0.10 & 0.05 \\
SAW & 0.69 & 0.36 & 40.6 & -0.12 & 0.09 & 0.09 & 0.13 & 0.03 \\
\hline
\end{tabular}

There were seven free parameters for five masking functions (Fig. 2) for each observer. Note that the negative values of the free parameter $\eta$ indicate that the weight of monoptic masking decreases with stimulus duration for all three observers.

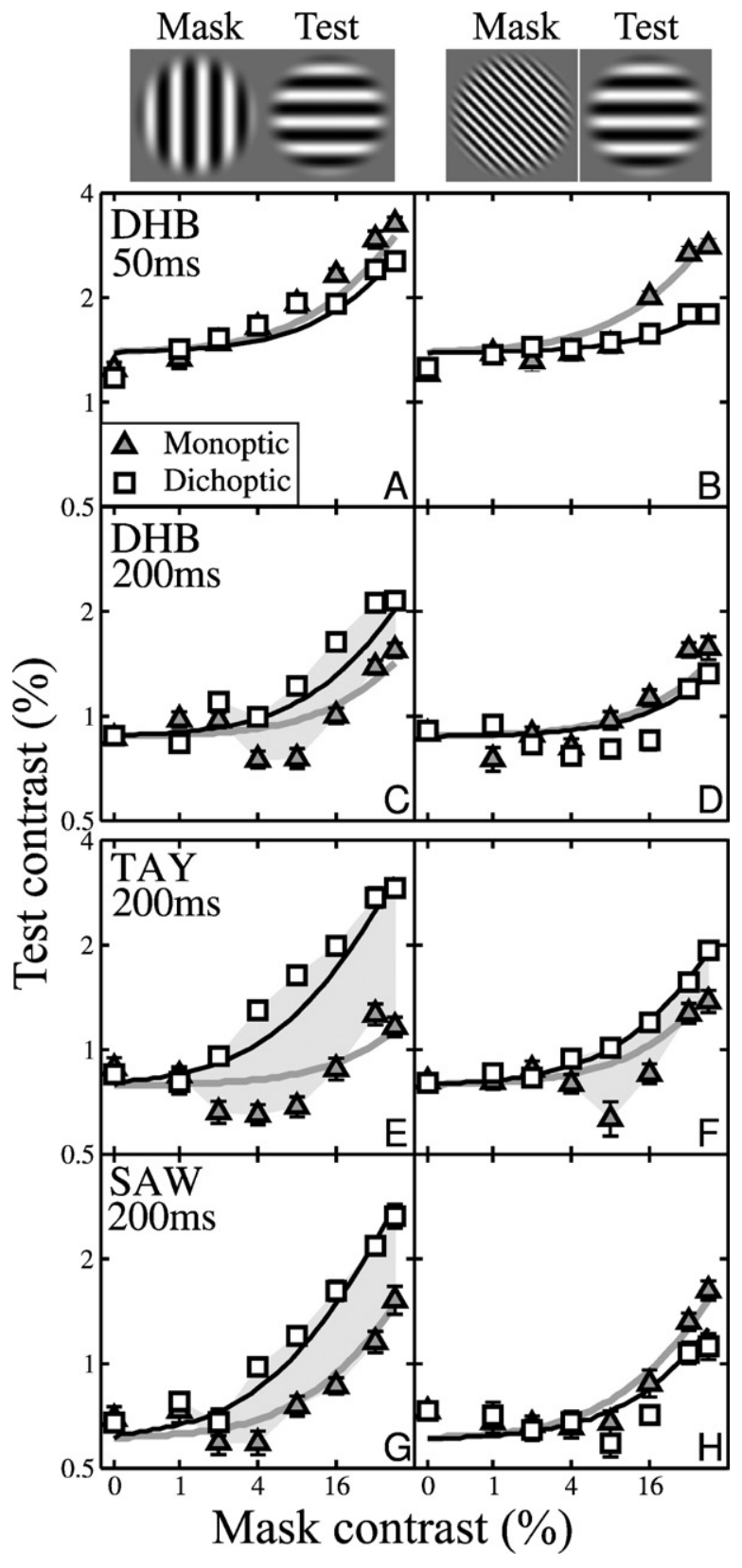

Fig. 5. Detection thresholds as functions of mask contrast. The shading indicates the regions where dichoptic $X O M>$ monoptic XOM. Error bars show $\pm 1 \mathrm{~S}$.E. of the estimates of threshold. Model predictions were generated using the parameters from experiment $\mathrm{I}$, with a single free sensitivity parameter $k$.
To test whether the order of entire contrast-masking functions (monoptic verses dichoptic) could be switched by stimulus duration, DHB repeated the experiment at $50 \mathrm{~ms}$ (Fig. 5a, b). As expected from Experiment I (Fig. 2, top panels), the switch occurred for the orthogonal mask, but not the oblique $3 \mathrm{c} /{ }^{\circ}$ mask (compare shaded regions in Fig. $5 a-d$, which shows where the dichoptic effect is greater than the monoptic effect).

Although the order of masking functions in Fig. 5 is diverse (compare shaded regions), a comparison of the results across the left and right panels shows that monoptic masking (triangles) is very similar for the two mask types. In contrast, dichoptic masking (squares) is weaker for the oblique $3 \mathrm{c} /{ }^{\circ}$ mask than for the orthogonal $1 \mathrm{c} /{ }^{\circ}$ mask. These effects are seen more directly in Fig. 6, which shows normalized detection thresholds in the presence of the 1 and $3 \mathrm{c} /{ }^{\circ}$ masks for the monoptic (left) and dichoptic (right) results from experiments I (top) and II (bottom). In both experiments there is little or no effect of mask type for the monoptic conditions (the lines cluster around horizontal

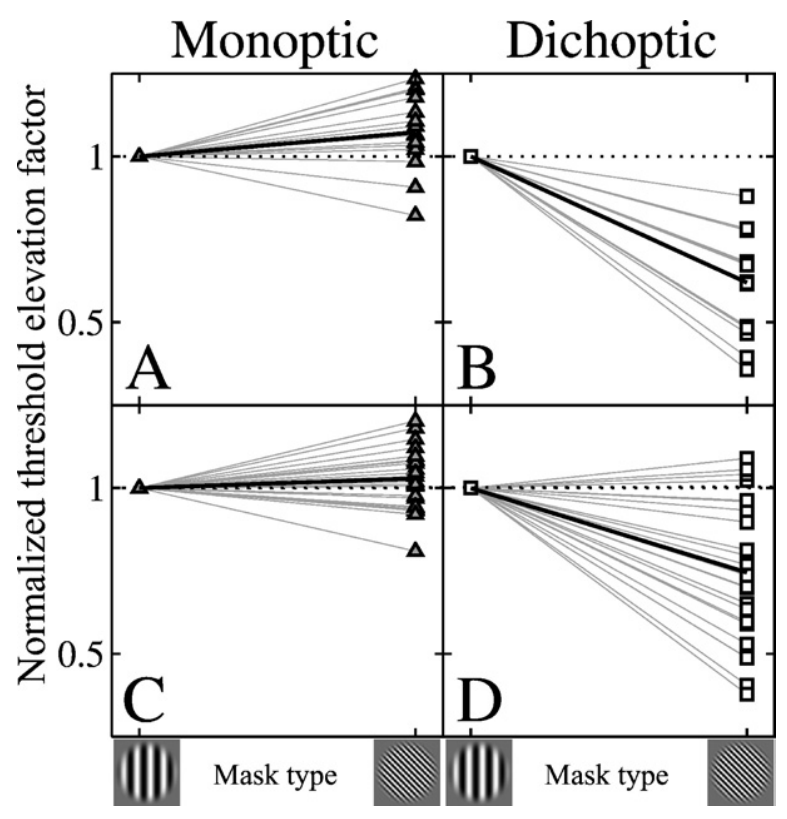

Fig. 6. Effect of mask type on threshold elevation factor for monoptic (left) and dichoptic (right) XOM. Data are collapsed across observer and stimulus duration in the top panels (experiment I; Fig. 2) and observer and mask contrast in the bottom panels (experiment II; Fig. 5). Threshold elevation was determined by calculating the ratio of contrast detection threshold with and without a mask. These functions were then normalized to unity for the $1 \mathrm{c} /{ }^{\circ}$ (vertical) mask. The solid lines are the average effects in each panel. The horizontal dotted lines indicate the locus of null effect. 


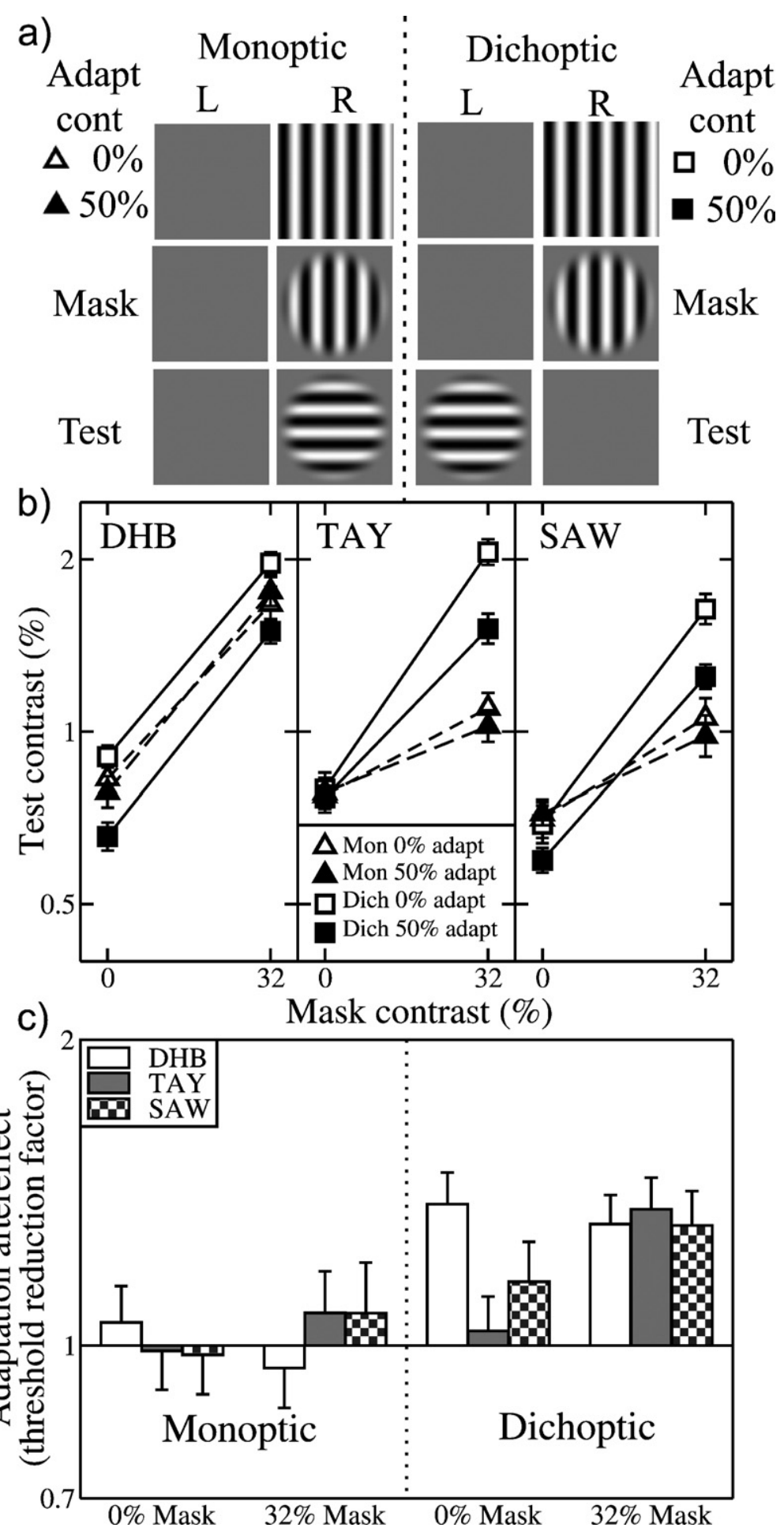

Fig. 7. Adapting the mask. Experimental design (a) thresholds (b) and adaptation aftereffects (c) for combined adaptation and cross-oriented masking at a stimulus duration of $100 \mathrm{~ms}$. The mask grating contrast was either $0 \%$ or $32 \%$ and the contrast of the adapting grating was either $0 \%$ or $50 \%$. Error bars show $\pm 1 \mathrm{~S}$.E. of the estimates of threshold. The threshold reduction factors in (c) were derived from the results in (b) by calculating the ratio of the detection thresholds for the null $(0 \%)$ and high contrast $(50 \%)$ adapters. A positive reduction factor indicates that the adapter decreased the potency of the mask.

in the panels on the left). However, the $3 \mathrm{c} /{ }^{\circ}$ mask is typically less effective than the $1 \mathrm{c} /{ }^{\circ}$ mask for the dichoptic conditions (most of the lines have negative gradients in the panels on the right). These observations are also confirmed by the monoptic and dichoptic weight parameters, $\omega_{M}$ and $\omega_{D}$ fitted by the model and shown in Table 1. Thus, 
Table 2. Quality of fit (RMS error) and parameter values for the modeling in experiment II

\begin{tabular}{llllllll}
\hline Observer & $\begin{array}{l}\text { Stimulus duration } \\
(\text { dur } \mathrm{ms})\end{array}$ & $\begin{array}{l}\text { RMS error } \\
(\mathrm{dB})\end{array}$ & $k$ & $\begin{array}{l}\omega_{M}(\text { dur }) \\
\left(1 \mathrm{c} /{ }^{\circ} \text { mask }\right)\end{array}$ & $\begin{array}{l}\omega_{M}(\text { dur }) \\
\left(3 \mathrm{c} /{ }^{\circ} \text { mask }\right)\end{array}$ & $\begin{array}{l}\omega_{D}(\text { dur }) \\
\left(1 \mathrm{c} /{ }^{\circ} \text { mask }\right)\end{array}$ & $\begin{array}{l}\omega_{D}(\text { dur }) \\
\left(3 \mathrm{c} /{ }^{\circ} \text { mask }\right)\end{array}$ \\
\hline DHB & 50 & 1.49 & 0.85 & 0.04 & 0.04 & 0.03 & 0.01 \\
DHB & 200 & 2.12 & 0.59 & 0.02 & 0.02 & 0.04 & 0.01 \\
TAY & 200 & 2.21 & 0.43 & 0.01 & 0.03 & 0.10 & 0.04 \\
SAW & 200 & 1.9 & 0.17 & 0.05 & 0.05 & 0.13 & 0.03 \\
\hline
\end{tabular}

There was one free parameter ( $k$ ) for four masking functions (Fig. 3) for each observer. Other model parameters were set to those shown in Table 1. The values of the weight functions $(\omega)$ are also shown for the appropriate stimulus durations. Much of the error (third column) is due to the facilitation in the monoptic condition, which was beyond the scope of the present model.

the ipsiocular route to XOM is broadband, whereas the interocular route is more narrowly tuned to spatial frequency, orientation or both.

Overall, there were small shifts in contrast sensitivity across experiments I and II so the model sensitivity parameter, k, was re-estimated to improve the fit in Fig. 5. This single free parameter served merely to shift the offset of the entire set of predictions for each observer, and did not change the order or their shape. The model captures the main features in the data very well (see curves in Fig. 5) confirming our earlier conclusions. The close relation between model predictions and data also confirms that the individual differences seen in experiment I were replicated in experiment II.

One shortcoming of the model is its failure to describe the small levels of facilitation at intermediate mask contrasts in some of the monoptic masking functions. This has been observed in other studies of XOM (Foley and Chen, 1997; Meese and Holmes, 2007; Meese et al., in press), but for simplicity is not modeled here. One way in which psychophysical facilitation can occur is if the mask gently stimulates the detecting mechanism bringing it closer to detection threshold (Legge and Foley, 1980). However, this process is typically associated with a reduction of the slope of the psychometric function (to a Weibull $\beta \sim 1.25$ ) (Pelli, 1985; Legge et al., 1987; Bird et al., 2002; Meese et al., 2006), whereas the psychometric functions here (not shown) were affected only modestly, if at all, by mask contrast and remained steep (typical Weibull $\beta \sim 3$ or 4 ). We have proposed an alternative account of this facilitatory effect elsewhere (Meese and Holmes, 2007; Meese et al., in press).

\section{Experiment III: adapting the mask}

Next we used an adaptation paradigm to establish the anatomical origins of the two routes to XOM. Because cortical cells are desensitized by prolonged stimulus exposure (Movshon and Lennie, 1979; Albrecht et al., 1984; Ohzawa et al., 1985), adaptation to the mask should decrease the effects of XOM if its site is cortical. A null effect would point to a pre-cortical locus because those cells are less prone to adaptation (Movshon and Lennie, 1979; Ohzawa et al., 1985; though see Shou et al., 1996; Solomon et al., 2004).

The mask had the same orientation as the adapter and was always presented to the same eye as the adapter. The test was presented to either the same eye as the mask and adapter (monoptic) or to the other eye (dichoptic) (see Fig. 7a). In the monoptic condition (triangles, Fig. 7b) the strength of masking was unaffected by adaptation to the mask (the dashed lines are very similar). However, when the test was presented to the other eye (squares, Fig. 7b), adaptation decreased the potency of the mask (the solid lines do not superimpose). These results are summarized in Fig. 7c, which shows the adaptation aftereffects for the monoptic (left) and dichoptic (right) conditions. Clearly, the aftereffects are much greater in the dichoptic condition, there being little or no aftereffect at all for monoptic stimulation.

The logic of our experiment was that if the suppression process were adaptable, then the potency of the mask would be reduced. For dichoptic masking, this happened for all three observers (far right of Fig. 7c). However, for DHB and SAW, adaptation to one eye enhanced sensitivity to orthogonal gratings in the other eye, even without the mask (left part of the dichoptic panel in Fig. 7c). This could be due to standing levels of interocular XOS in the cortex (Morrone et al., 1982) that are diminished by the adaptation process (De Valois, 1977; Tolhurst and Barfield, 1978; Greenlee and Magnussen, 1988). If so, then in this experiment the differences between the observers are understood as individual differences in (standing) suppressive weights, along similar lines as before (Tables 1 and 2). Another possibility is that adaptation does not desensitize the process of suppression, but provides direct facilitation for orthogonal gratings in the other eye. However, this interpretation is difficult to reconcile with the results for TAY where the dichoptic aftereffect was restricted to the high contrast mask condition (see Fig. 7c).

The results of this adaptation experiment suggest a sequence to the two forms of masking: a pre-cortical monoptic process, followed by a cortical dichoptic process. In the model, the effects are achieved by allowing only the interocular pathway for XOS to be desensitized by prolonged stimulation (i.e. only $\omega_{D}$ decreases with adaptation in Fig. 1d).

\section{DISCUSSION}

We have considered four arrangements (models) for the possible loci of ipsiocular and interocular XOS relative to binocular summation. Model 1 places suppression from both eyes after binocular summation and predicts that 
contrast-masking functions should be identical for monoptic and dichoptic masks (Fig. 1a). This model is rejected by the masking results from all three of our experiments where we find no evidence for superposition. Models 2 and 3 are extensions of Model 1 where the late suppression is augmented by either an ipsiocular (Model 2) or interocular (Model 3) pathway. These models predict that one form of masking should be consistently greater than the other (Fig. 1b and c). Both models are rejected by the results from experiments I and II, where we find that the order of the effects depends upon stimulus duration, spatial configuration of the mask, and observer. The only arrangement with sufficient flexibility to accommodate the complexity of our results is Model 4, where both sources of masking are placed before binocular summation (Fig. 1d).

These psychophysical results and quantitative systems analyses provide the first clear evidence for two stages of superimposed XOM in human vision. This work extends our earlier model of binocular, monoptic and dichoptic pedestal masking (Meese et al., 2006) to the more general case of XOM (though for simplicity, we have restricted the present implementation to operate only around detection threshold).

\section{Cortical and pre-cortical sources of XOS}

The ipsiocular effect was spatially broadband, its suppressive weight tended to decrease with stimulus duration (Table 1) and it was not adaptable. These characteristics are consistent with pre-cortical neurons but not those typical of the cortex. For example, recent work in the LGN of cat found non-classical suppression that extends over the entire receptive field and was broadly tuned to spatial frequency (Bonin et al., 2005). XOS in cat is most pronounced at high temporal frequencies (Li et al., 2005), consistent with the decline of our ipsiocular effect with stimulus duration. And it is well known that cortical cells adapt, but that cells in the LGN do not (Movshon and Lennie, 1979; Ohzawa et al., 1985), at least under conventional stimulation (Solomon et al., 2004).

In contrast, we found that the weight of interocular masking increased with stimulus duration, consistent with the finding that interocular XOS is greatest for low temporal frequencies at the single-cell level in cat (Li et al., 2005). A striking result here is that the functional form of this temporal dependency of XOS is identical to that of contrast sensitivity to the test grating (see the subsection on model details), implying that contrast detection and interocular XOS follow identical, presumably cortical, stages of temporal integration (Legge, 1978; Georgeson, 1987). Our finding of orientation and/or spatial frequency tuning for interocular XOM also points to a cortical origin (Hubel and Wiesel, 1959; Maffei and Fiorenti, 1973; Movshon et al., 1978). Further support for this hypothesis comes from our adaptation study, where dichoptic XOM was diminished by mask adaptation, consistent with cortical physiology (Movshon and Lennie, 1979; Albrecht et al., 1984; Carandini et al., 1998). Analogous adaptation experiments at the single-cell level in cat also suggest pre-cortical and cortical involvement for XOS within and between the eyes respectively (Freeman et al., 2002; Li et al., 2005; Sengpiel and Vorobyov, 2005). Finally, our previous work on binocular XOM (Meese and Hess, 2004; Meese and Holmes, 2007) shows that it is a low spatial frequency and high temporal frequency phenomenon; a result that we have recently replicated for monoptic stimuli (T. S. Meese and D. H. Baker, unpublished observations). Thus, the pre-cortical form of XOS might be associated most strongly with the magnocellular stream in primate.

Our work above addresses the sequence of origin for the two sources of suppression, but it does not address the sequence of their influence. For simplicity, the illustration of Model 4 (Fig. 1d) shows a common impact site for both sources of XOS, but for the stimulus conditions described so far this arrangement shares a formal similarity with one in which they are placed in series. Therefore, it is possible that (i) the two sources of suppression affect the signal in the anatomical sequence in which they originate, or (ii) the cortical influence feeds back to the LGN to impact at a common site. We now present evidence for the former alternative.

\section{Interocular suppression of binocular masks}

When a mask is presented to both eyes, one might suppose that suppression from both of the cross-oriented pathways is of consequence (see Fig. 1d and Eq. 2) and that masking would increase. However, other work indicates that the characteristics of binocular XOM are much closer to monoptic than dichoptic XOM. Both are immune to adaptation (Foley and Chen, 1997), both are spatially broadband (Meese, 2004), and both are most pronounced at low spatial frequencies and high temporal frequencies or short durations (Meese and Holmes, 2007). This apparent paradox could occur if the parallel masks in each eye were to suppress each other (interocular suppression) before their own binocular summation, thereby reducing the effective mask contrast available for dichoptic XOM (Meese and Hess, 2005). Indeed, this arrangement goes some way toward explaining why the contrast of the world does not change very much when you view it with two eyes instead of one: the extra contrast from the second eye is offset by the interocular suppression it imposes on the first eye (Ding and Sperling, 2006; Meese et al., 2006; Baker et al., in press).

We tested this idea in an extension to the present study by adding a second mask to what was originally a dichoptic (or monoptic) mask. We call this a 'half-binocular' condition because the cross-oriented mask is shown to both eyes, but the test grating to only one (Meese and Hess, 2005; Meese et al., 2006). Results for this condition (diamonds) are shown in Fig. 4 along with those from the monoptic (triangles) and dichoptic (squares) conditions replotted from Fig. 2. Where dichoptic XOM dominates (shading in Fig. 4), half-binocular masking was typically less effective than dichoptic masking (filled diamonds), even though there was greater mask energy across the eyes (McKee et al., 1994; Meese and Hess, 2005). This can be understood by supposing that the extra (monoptic) mask in the half- 
binocular condition activates interocular suppression between the two mask components, thereby weakening the otherwise powerful dichoptic XOM. Although the extra monoptic mask also adds to the overall level of suppression through the ipsiocular pathway, its weak effect in these conditions is insufficient to overcome the reduction of dichoptic XOM. Furthermore, if the consequence of interocular suppression does not feed back to the LGN (i.e. the process of monoptic masking is beyond the reach of interocular effects), then monoptic XOM will not be released in the same way. This is evident in Fig. 4 in the regions where monoptic XOM dominates (triangles >squares; no shading). In those cases the extra mask typically enhances masking (open diamonds), because of the additional interocular effect (see figure caption for further details). This implies that the two forms of masking exert their influence in the same order in which they originate.

We conclude that XOS occurs at (i) a pre-cortical site within purely monoptic pathways, and (ii) at a subsequent cortical stage between the eyes, and before full binocular summation of signals (Fig. 8). As visual area 1 (V1) is the first stage at which signals are summed across eyes, and as most cells in higher visual areas are also binocular (Leopold and Logothetis, 1996), the most likely site for our cortical stage of interocular suppression, is V1. However, we emphasize that the anatomical assignments (either side of the dashed line in Fig. 8) come from the association between our psychophysical results and the known properties of cortical and pre-cortical neurons. Thus, if a subgroup of cells in V1 (e.g. in layer 4), were to share the crucial properties of cells in the LGN, we could not rule out

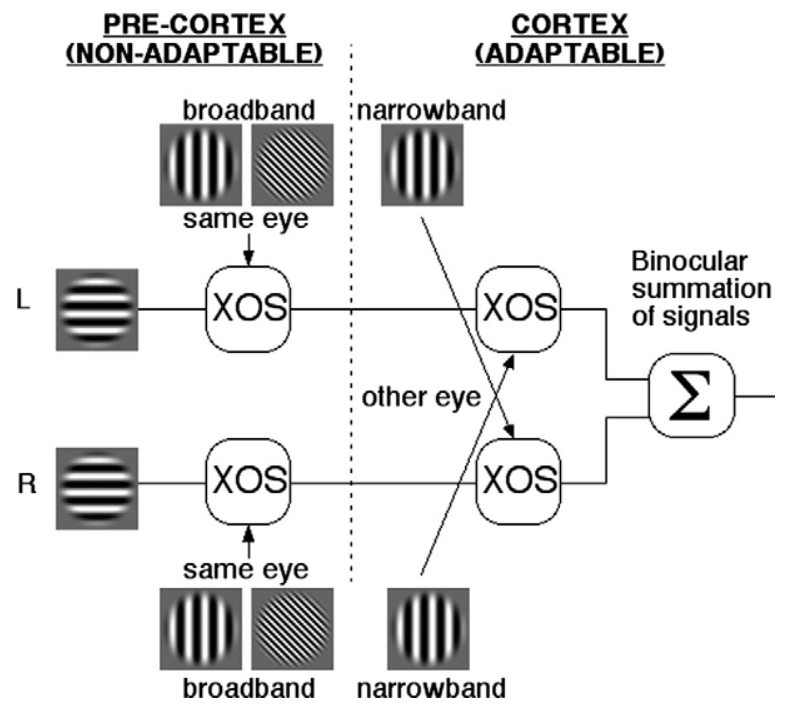

Fig. 8. Schematic illustration of the anatomical arrangement of XOS implied by our experiments. The broadband and narrowband origins of XOS are not thought to be limited to the orientations and spatial frequencies depicted by the icons. Our experiments do not rule out an ipsiocular contribution to XOS at the cortical stage or further interactions after binocular summation. The second stage of suppression might involve interactions between ocular-dominated cells rather than those with purely monoptic drive as depicted. the possibility that the broadband ipsiocular effects reside there (see also Smith et al., 2006; Webb et al., 2005).

The logic in Fig. 1 is developed around an architecture in which purely monoptic pathways (in terms of excitation) feed directly into full binocular pathways capable of substantial binocular summation (see Appendix A). A more likely arrangement is that an intermediate stage has intermediate levels of binocular summation consistent with left and right ocular dominances (Hubel and Wiesel, 1959). The logic of our approach also applies in that situation, in which case the binocular site in Figs. 1 and 8 represents the later stage of full binocular summation. Thus, it is possible that our second stage of XOS occurs between ocular-dominated cells, rather than cells with purely monoptic drive.

\section{Implications and comparison with other studies}

Single-cell studies of dichoptic XOS have typically reported results for binocular cells (Walker et al., 1998; Freeman et al., 2002; Li et al., 2005; Sengpiel and Vorobyov, 2005). Within our scheme (Fig. 8), dichoptic XOS will be apparent in full binocular cells, but it should also be found among cells with purely monoptic drive, or at least those dominated by drive from one eye (as discussed above). We know of no experiments that have addressed this.

Although there are close comparisons between our psychophysical experiments on humans and single-cell work in cat (see above), little related work has been done in monkey (though see Smith et al., 2006) and analogous experiments have not been performed. Other work performed by Macknik and his colleagues (Tse et al., 2005; Macknik and Martinez-Conde, 2004) used a very different type of stimulus in which the mask and test contained similar oriented contours, but were not superimposed in either space or time. In an imaging study on humans (Tse et al., 2005), and a single-cell study on monkey (Macknik and Martinez-Conde, 2004), Macknik drew the opposite conclusion from us: that dichoptic influences arise after binocular summation. Our scheme (Fig. 8) does not rule out the possibility of further interactions beyond binocular summation and the lateral inhibitory interactions associated with Macknik's stimuli (Tse et al., 2005; Macknik and Martinez-Conde, 2004) are a strong candidate for this. Certainly, their finding of identical psychophysical effects for binocular and dichoptic masking sets their result apart from other studies that have used superimposed (Legge, 1979; Levi et al., 1979; Meese et al., 2006) or annular (Meese and Hess, 2004; Petrov and McKee, 2006) gratings.

A two-stage sequence for suppression has also been identified psychophysically for (i) superimposed XOM and (ii) masking from co-oriented gratings in the surround (Petrov et al., 2005). It seems likely that the first stages identified by us and by Petrov et al. (2005) involve a common mechanism (though here we also provide anatomical context), but whether the second stages involve different mechanisms is not clear (Meese and Hess, 2004; Petrov and McKee, 2006). Thus, the possibility of at least three different processes for masking by suppression re- 
mains viable in humans as well as in cats (Sengpiel et al., 1998). (See also Webb et al., 2005.)

XOS is a well-established component (Foley, 1994) in image-processing models of spatial vision, which aim to specify the information processing capacities of the early visual system. Hitherto, cross-orientation interactions have been expressed at a single stage (Watson and Solomon, 1997). It is now clear that at least two stages with different spatio-temporal characteristics are needed to provide a more complete representation of image coding in human vision.

\section{Binocular rivalry}

The stimuli used in studies of XOS are similar to those frequently used in studies of another, presumably related form of suppression (Sengpiel et al., 1995; Harrad, 1996; Brown et al., 1999) known as binocular rivalry. In a typical experiment, very different images (e.g. orthogonal gratings) are presented to different eyes, and after a second or so they start to rival: for brief periods the image in either the left or right eye dominates, or a mixture of the two is seen, with different eyes dominating in different parts of the image (Lee and Blake, 2004). Binocular rivalry has traditionally been attributed to ocular interactions in V1 (Blake, 1989), but single-cell recordings (Leopold and Logothetis, 1996), functional imaging (Tse et al., 2005) and behavioral studies (Kovacs et al., 1996; Logothetis et al., 1996) have painted a more complex picture (see Freeman et al. (2005) for a review). One view is that rivalry is image-based rather than eye-based (Kovacs et al., 1996; Logothetis et al., 1996), though recent work has found that whatever the drive, the consequences can be observed as early as the LGN (Haynes et al., 2005; Wunderlich et al., 2005). Notwithstanding the involvement of higher-order interactions (Leopold and Logothetis, 1996; Logothetis et al., 1996; Logothetis, 1998; Macknik and Martinez-Conde, 2004; Tse et al., 2005), it now seems likely that a substantial contribution to binocular rivalry arises from interocular suppression (Sengpiel et al., 1995; Harrad, 1996; Wilson, 2003; Lee and Blake, 2004; Freeman et al., 2005). Nevertheless, it has been difficult to determine the origins of the effects observed in various brain areas within either functional or anatomical maps (Polonsky et al., 2000; Haynes et al., 2005; Lee et al., 2005; Tse et al., 2005; Wunderlich et al., 2005). Typical behavioral experiments on binocular rivalry are not a good starting point for this task because the inherently subjective nature of rivalry (Freeman et al., 2005) means that criterion-dependent behavioral results do not constrain a formal systems-analysis. A psychophysical approach much better suited to the modeler's needs is the objective measurement of visual performance using two-interval forced-choice. Our experiments here provide this formality and strong evidence for ocular and spatial interactions in the early visual system. The short stimulus durations and low target contrasts that we used were designed to produce strong suppressive effects, but no rivalry. Thus, while our experiments were not intended to tap binocular rivalry itself, they do provide new information on the biological hardware that is available to support it. In fact, the interocular pathway identified here is a plausible candidate for the initial stage of eye-based interactions for cross-oriented stimuli in recent hierarchical models of binocular rivalry (Wilson, 2003; Freeman et al., 2005).

\section{Diplopia suppression?}

One possible functional role for these interocular interactions is the suppression of diplopia (double vision). Because the two eyes have slightly different views on the world, the retinal images are not identical under normal viewing, and cannot be slid into register. For objects whose binocular disparity is within 'Panum's fusional area' this does not present a problem because the visual system can tolerate some misalignment of the two monoptic images, and uses it to calculate depth (stereopsis) (see Howard, 2002 for review). However, in some situations a single object projects to substantially disparate locations on the retinae and therefore cannot be fused. A corollary of this is that different objects (or their parts) can project to corresponding regions on the two retinae, causing different stimulus orientations to superimpose in the binocular image, in just the same way as for our dichoptic stimuli. (Direct inspection of almost any red/green anaglyph of a natural scene will confirm the point: regions can be found where red and green contours superimpose at different orientations.)

This unfortunate consequence of geometry raises at least two problems for the binocular visual system: (i) images with large disparities do not fuse, leading to diplopia, and (ii) the superposition of non-fused regions is likely to produce intersecting contours in binocular perception (one from each eye), even though they do not exist in the distal stimulus or in either retinal image. Clearly, a solution to either of these problems will help solve the other. We suggest that interocular XOS would help solve the second problem by suppressing the weaker of the two features around the perceived intersection. However, the process is not automatic, but influenced by the matching of corresponding features across the eyes (McKee et al., 1994; Grossberg and Howe, 2003; Meese and Hess, 2005), as in the half-binocular condition here. Indeed, when this occurs (as for binocular masks), then it might be taken as evidence that corresponding retinal points are seeing a single object, in which case dichoptic XOS should be diminished, just as we found.

Acknowledgments-This work was supported by grants from the Wellcome Trust (069881/Z/O2/Z) awarded to Tim Meese and the Engineering and Physical Sciences Research Council (GR/ S74515/01) awarded to Tim Meese and Mark Georgeson. We would also like to thank two anonymous reviewers for their constructive comments.

\section{REFERENCES}

Albrecht DG, Farrar SB, Hamilton DB (1984) Spatial contrast adaptation characteristics of neurons recorded in the cat's visual-cortex. J Physiol Lond 347:713-739.

Baker DH, Meese TS, Georgeson MA (2007) Binocular interaction: Contrast matching and contrast discrimination are predicted by the same model. Spatial Vision, in press. 
Bird CM, Henning GB, Wichmann FA (2002) Contrast discrimination with sinusoidal gratings of different spatial frequency. J Opt Soc Am A Opt Image Sci Vis 19:1267-1273.

Blake R (1989) A neural theory of binocular-rivalry. Psychol Rev 96:145-167.

Bonds $A B$ (1989) Role of inhibition in the specification of orientation selectivity of cells in the cat striate cortex. Vis Neurosci 2:41-55.

Bonin V, Mante V, Carandini M (2005) The suppressive field of neurons in lateral geniculate nucleus. J Neurosci 25:10844-10856.

Born RT, Tootell RBH (1991) Single-unit and 2-deoxyglucose studies of side inhibition in macaque striate cortex. Proc Natl Acad Sci U S A 88:7071-7075.

Brown RJ, Candy TR, Norcia AM (1999) Development of rivalry and dichoptic masking in human infants. Invest Ophthalmol Vis Sci 40:3324-3333.

Carandini M, Movshon JA, Ferster D (1998) Pattern adaptation and cross-orientation interactions in the primary visual cortex. Neuropharmacology 37:501-511.

De Valois KK (1977) Spatial frequency adaptation can enhance contrast sensitivity. Vision Res 23:249-256.

Ding J, Sperling G (2006) A gain-control theory of binocular combination. Proc Natl Acad Sci U S A 103:1141-1146.

Felisberti F, Derrington AM (1999) Long-range interactions modulate the contrast gain in the lateral geniculate nucleus of cats. Vis Neurosci 16:943-956.

Foley JM (1994) Human luminance pattern-vision mechanisms: masking experiments require a new model. J Opt Soc Am A Opt Image Sci Vis 11:1710-1719.

Foley JM, Chen CC (1997) Analysis of the effect of pattern adaptation on pattern pedestal effects: A two-process model. Vision Res $37: 2781-2788$.

Freeman AW, Nguyen VA, Alais D (2005) The nature and depth of binocular rivalry suppression. In: Binocular rivalry (Alais D, Blake R, eds), pp 47-62. MIT Press: London.

Freeman TCB, Durand S, Kiper DC, Carandini M (2002) Suppression without inhibition in visual cortex. Neuron 35:759-771.

Georgeson MA (1987) Temporal properties of spatial contrast vision. Vision Res 27:765-780.

Georgeson MA, Meese TS (2005) Binocular summation at contrast threshold: A new look. Perception 34:138-138.

Greenlee MW, Magnussen S (1988) Interactions among spatial-frequency and orientation channels adapted concurrently. Vision Res 28:1303-1310.

Grossberg S, Howe PDL (2003) A laminar cortical model of stereopsis and three-dimensional surface perception. Vision Res 43:801-829.

Harmon LD, Julesz B (1973) Masking in visual recognition: effects of 2-dimensional filtered noise. Science 180:1194-1197.

Harrad R (1996) Psychophysics of suppression. Eye 10:270-273.

Haynes JD, Deichmann R, Rees G (2005) Eye-specific effects of binocular rivalry in the human lateral geniculate nucleus. Nature 438:496-499.

Heeger DJ (1992) Normalization of cell responses in cat striate cortex. Vis Neurosci 9:181-197.

Howard IP (2002) Seeing in depth: Basic mechanisms. Ontario: I Porteous.

Hubel DH, Wiesel TN (1959) Receptive fields of single neurones in the cat's visual cortex. J Physiol 148:574-591.

Kovacs I, Papathomas TV, Yang M, Feher A (1996) When the brain changes its mind: Interocular grouping during binocular rivalry. Proc Natl Acad Sci U S A 93:15508-15511.

Lee SH, Blake R (2004) A fresh look at interocular grouping during binocular rivalry. Vision Res 44:983-991.

Lee SH, Blake R, Heeger DJ (2005) Traveling waves of activity in primary visual cortex during binocular rivalry. Nat Neurosci 8:22-23.

Legge GE (1978) Sustained and transient mechanisms in humanvision: temporal and spatial properties. Vision Res 18:69-81.
Legge GE (1979) Spatial-frequency masking in human-vision: binocular interactions. J Opt Soc Am 69:838-847.

Legge GE, Foley JM (1980) Contrast masking in human-vision. J Opt Soc Am 70:1458-1471.

Legge GE, Kersten D, Burgess AE (1987) Contrast discrimination in noise. J Opt Soc Am A Opt Image Sci Vis 4:391-404.

Leopold DA, Logothetis NK (1996) Activity changes in early visual cortex reflect monkeys' percepts during binocular rivalry. Nature 379:549-553.

Levi DM, Harwerth RS, Smith EL (1979) Humans deprived of normal binocular vision have binocular interactions tuned to size and orientation. Science 206:852-854.

Levick WR, Cleland BG, Dubin MW (1972) Lateral geniculate neurons of cat: retinal inputs and physiology. Invest Ophthalmol 11:302.

Li BW, Peterson MR, Thompson JK, Duong T, Freeman RD (2005) Cross-orientation suppression: monoptic and dichoptic mechanisms are different. J Neurophysiol 94:1645-1650.

Li BW, Thompson JK, Duong T, Peterson MR, Freeman RD (2006) Origins of cross-orientation suppression in the visual cortex. J Neurophysiol 96:1755-1764.

Logothetis NK (1998) Single units and conscious vision. Philos Trans R Soc Lond B Biol Sci 353:1801-1818.

Logothetis NK, Leopold DA, Sheinberg DL (1996) What is rivalling during binocular rivalry? Nature 380:621-624.

Luntinen O, Rovamo J, Nasanen R (1995) Modelling the increase of contrast sensitivity with grating area and exposure time. Vision Res 35:2339-2346.

Macknik SL, Martinez-Conde S (2004) Dichoptic visual masking reveals that early binocular neurons exhibit weak interocular suppression: implications for binocular vision and visual awareness. J Cogn Neurosci 16:1049-1059.

Maffei L, Fiorenti A (1973) Visual-cortex as a spatial frequency analyzer. Vision Res 13:1255-1267.

McKee SP, Bravo MJ, Taylor DG, Legge GE (1994) Stereo matching precedes dichoptic masking. Vision Res 34:1047-1060.

Meese TS (2004) Area summation and masking. J Vision 4:930-943.

Meese TS, Georgeson MA, Baker DH (2006) Binocular contrast vision at and above threshold. J Vision 6:1224-1243.

Meese TS, Hess RF (2004) Low spatial frequencies are suppressively masked across spatial scale, orientation, field position, and eye of origin. J Vision 4:843-859.

Meese TS, Hess RF (2005) Interocular suppression is gated by interocular feature matching. Vision Res 45:9-15.

Meese TS, Holmes DJ (2002) Adaptation and gain pool summation: alternative models and masking data. Vision Res 42:1113-1125.

Meese TS, Holmes DJ (2007) Spatial and temporal dependencies of cross-orientation suppression in human vision. Proc $\mathrm{R}$ Soc Lond $\mathrm{B}$ Biol Sci 274:127-136.

Meese TS, Summers RJ, Holmes DJ, Wallis SA (2007) Contextual modulation involves suppression and facilitation from the centre and the surround. J Vision, in press.

Morrone MC, Burr DC, Maffei L (1982) Functional implications of cross-orientation inhibition of cortical visual cells. 1. Neurophysiological evidence. Proc R Soc Lond B Biol Sci 216:335-354.

Morrone MC, Burr DC, Ross J (1983) Added noise restores recognizability of coarse quantized images. Nature 305:226-228.

Morrone MC, Burr DC, Speed HD (1987) Cross-orientation inhibition in cat is GABA mediated. Exp Brain Res 67:635-644.

Movshon JA, Lennie P (1979) Pattern-selective adaptation in visual cortical-neurons. Nature 278:850-852.

Movshon JA, Thompson ID, Tolhurst DJ (1978) Spatial summation in receptive-fields of simple cells in cats striate cortex. J Physiol Lond 283:53-77.

Murphy PC, Sillito AM (1989) The binocular input to cells in the feline dorsal lateral geniculate-nucleus (DLGN). J Physiol Lond 415: 393-408.

Ohzawa I, Sclar G, Freeman RD (1985) Contrast gain-control in the cats visual-system. J Neurophysiol 54:651-667. 
Olzak LA, Thomas JP (1999) Neural recoding in human pattern vision: model and mechanisms. Vision Res 39:231-256.

Pelli DG (1985) Uncertainty explains many aspects of visual contrast detection and discrimination. J Opt Soc Am A Opt Image Sci Vis 2:1508-1532.

Pelli DG (1987) On the relation between summation and facilitation. Vision Res 27:119-123.

Petrov Y, Carandini M, McKee S (2005) Two distinct mechanisms of suppression in human vision. J Neurosci 25:8704-8707.

Petrov Y, McKee S (2006) The effect of spatial configuration on surround suppression of contrast sensitivity. J Vision 6:224-238.

Polonsky A, Blake R, Braun T, Heeger DJ (2000) Neuronal activity in human primary visual cortex correlates with perception during binocular rivalry. Nat Neurosci 3:1153-1159.

Priebe NJ, Ferster D (2006) Mechanisms underlying cross-orientation suppression in cat visual cortex. Nat Neurosci 9:552-561.

Sanderson KJ, Darian-Smith I, Bishop PO (1969) Binocular corresponding receptive fields of single units in the cat dorsal lateral geniculate nucleus. Vision Res 9:1297-1303.

Sengpiel F, Baddeley RJ, Freeman TCB, Harrad R, Blakemore C (1998) Different mechanisms underlie three inhibitory phenomena in cat area 17. Vision Res 38:2067-2080.

Sengpiel F, Blakemore C, Harrad R (1995) Interocular suppression in the primary visual-cortex: a possible neural basis of binocularrivalry. Vision Res 35:179-195.

Sengpiel F, Vorobyov V (2005) Intracortical origins of interocular suppression in the visual cortex. J Neurosci 25:6394-6400.

Shou TD, Li XG, Zhou YF, Hu B (1996) Adaptation of visually evoked responses of relay cells in the dorsal lateral geniculate nucleus of the cat following prolonged exposure to drifting gratings. Vis Neurosci 13:605-613.
Smith MA, Bair W, Movshon JA (2006) Dynamics of suppression in macaque primary visual cortex. J Neurosci 26:4826-4834.

Solomon JA, John A, Morgan MJ (2006) Monocular texture segmentation and proto-rivalry. Vision Res 46:1488-1492.

Solomon SG, Peirce JW, Dhruv NT, Lennie P (2004) Profound contrast adaptation early in the visual pathway. Neuron 42:155-162.

Tolhurst DJ (1975) Reaction times in the detection of gratings by human observers: A probabilistic mechanism. Vision Res 15: 1143-1149.

Tolhurst DJ, Barfield LP (1978) Interactions between spatial-frequency channels. Vision Res 18:951-958.

Tse PU, Martinez-Conde S, Schlegel AA, Macknik SL (2005) Visibility, visual awareness, and visual masking of simple unattended targets are confined to areas in the occipital cortex beyond human V1/V2. Proc Natl Acad Sci U S A 102:17178-17183.

Walker GA, Ohzawa I, Freeman RD (1998) Binocular cross-orientation suppression in the cat's striate cortex. J Neurophysiol 79:227-239.

Watson AB (1979) Probability summation over time. Vision Res 19:515-522.

Watson AB, Solomon JA (1997) Model of visual contrast gain control and pattern masking. J Opt Soc Am A Opt Image Sci Vis 14:2379-2391.

Webb BS, Dhruv NT, Solomon SG, Tailby C, Lennie P (2005) Early and late mechanisms of surround suppression in striate cortex of macaque. J Neurosci 25:11666-11675.

Wetherill GB, Levitt H (1965) Sequential estimation of points on a psychometric function. Br J Math Stat Psychol 18:1-10.

Wilson HR (2003) Computational evidence for a rivalry hierarchy in vision. Proc Natl Acad Sci U S A 100:14499-14503.

Wunderlich K, Schneider KA, Kastner S (2005) Neural correlates of binocular rivalry in the human lateral geniculate nucleus. Nat Neurosci 8:1595-1602.

\section{APPENDIX A}

\section{The transducer exponents can be derived from the psychometric function and the binocular summation ratio}

In the absence of masking, equation 1 reduces to:

$$
\operatorname{resp}_{\text {test }}=\left(L^{m}+R^{m}\right)^{p}
$$

where $L$ and $R$ are the test contrasts in the left and right eyes respectively. We now derive estimates of $m$ and $p$, using information about the slope of the psychometric function, and the binocular summation ratio.

The sigmoidal Weibull function provides a good fit to typical psychometric functions where its shape is controlled by a single parameter, $\beta$ (increasing $\beta$ makes it steeper). The geometric mean $(n=48)$ of the slope of the psychometric function $(\beta)$ was calculated for the monoptic no-mask condition in experiment I for the three observers, giving $\beta=4.37$. From signal detection theory there is a formal relation between the overall exponent of the contrast transducer $(b)$ and the log-log slope of the $d^{\prime}$ psychometric function, and from Pelli (1987) this extends to an approximation of the Weibull slope: $b \sim \beta / 1.25$, giving $b \sim 3.5$. In equation $\mathrm{A} 1, b$ is equal to the product of the two exponents, $m$ and $p$. The value of $m$ can be inferred directly from the binocular summation ratio $\left(B_{\text {sum }}\right)$, which is the ratio of monoptic and binocular detection thresholds $\left(B_{\text {sum }}=C_{\text {mon }} / C_{\text {bin }}\right)$. These were gathered in experiment II (not shown), from which we estimated $B_{\text {sum }}=1.7(n=32)$. Because sensitivity was the same for the two eyes, equation 1 tells us that $\left(2 C_{b i n}{ }^{m}\right)^{p}=\left(C_{m o n}{ }^{m}\right)^{p}$, and therefore that $B_{\text {sum }}=2^{1 / m}$. This means $m=1.3$, and $p=b / m=2.8$. These values of $m, p$ and $B_{\text {sum }}$ are consistent with our earlier and extensive work on this issue (Georgeson and Meese, 2005). 